UB-ECM-PF 95/11

April 1995

\title{
Phase structure of renormalizable four-fermion models in spacetimes of constant curvature
}

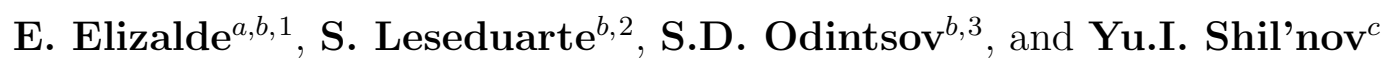 \\ ${ }^{a}$ Centre for Advanced Studies CEAB, CSIC, Camí de Santa Bàrbara, 17300 Blanes, Spain \\ ${ }^{b}$ Department ECM and IFAE, Faculty of Physics, University of Barcelona, \\ Diagonal 647, 08028 Barcelona, Spain \\ ${ }^{c}$ Department of Theoretical Physics, Kharkov State University, \\ Svobody Sq. 4, Kharkov 310077, Ukraine
}

\begin{abstract}
A number of $2 \mathrm{~d}$ and $3 \mathrm{~d}$ four-fermion models which are renormalizable - in the $1 / N$ expansion - in a maximally symmetric constant curvature space, are investigated. To this purpose, a powerful method for the exact study of spinor heat kernels and propagators on maximally symmetric spaces is reviewed. The renormalized effective potential is found for any value of the curvature and its asymptotic expansion is given explicitly, both for small and for strong curvature. The influence of gravity on the dynamical symmetry breaking pattern of some $\mathrm{U}(2)$ flavor-like and discrete symmetries is described in detail. The phase diagram in $S^{2}$ is constructed and it is shown that, for any value of the coupling constant, a curvature exists above which chiral symmetry is restored. For the case of $H^{2}$, chiral symmetry is always broken. In three dimensions, in the case of positive curvature, $S^{3}$, it is seen that curvature can induce a second-order phase transition. For $H^{3}$ the configuration given by the auxiliary fields equated to zero is not a solution of the gap equation.
\end{abstract}

PACS: 04.62.+v, 04.60.-m, 02.30.+g

\footnotetext{
${ }^{1}$ E-mail: eli@zeta.ecm.ub.es

${ }^{2}$ E-mail: lese@zeta.ecm.ub.es

${ }^{3}$ On leave of absence from Tomsk Pedagogical Institute, 634041 Tomsk, Russia. E-mail: odintsov@ecm.ub.es
} 


\section{Introduction}

Four-fermion models [1, 2] - usually considered in the $1 / N$ expansion - are interesting due to the fact that they provide the opportunity to carry out an explicit, analytical study of composite bound states and dynamical chiral symmetry breaking. At the same time, these theories - and specially their renormalizable 2d [2] and 3d [3] variants - exhibit specific properties which are similar to the basic behaviors of some realistic models of particle physics. Moreover, this class of theories can be used for the description of the standard model (SM) itself, or of some particle physics phenomena in the SM (see 团-[7]). For example, the dynamical symmetry breking pattern of Nambu-Jona-Lasinio (NJL) models for the electroweak interaction, with the top quark as an order parameter, has been discussed in 四, 司.

Having in mind the applications of four-fermion models to the early universe and, in particular, the chiral symmetry phase transitions that take place under the action of the external gravitational field, there has been recently some activity in the study of $2 \mathrm{~d}$ [8], 3d [9] and 4d [10]-14] four-fermion models in curved spacetime (for a general introduction to quantum field theory in a curved spacetime, see [15]). The block-spin renormalization group (RG) approach and the similarities of the model with the Higgs-Yukawa one have been considered in [13] and [14], respectively.

The effective potential of composite fermions in curved spacetime has been calculated in different dimensions [8]-11]. Dynamical chiral symmetry breaking, fermionic mass generation and curvature-induced phase transitions have been investigated in full detail. However, in most of these cases only the linear curvature terms of the effective potential have been taken into account [9]-[12]. But it turns out in practice that it is often necessary to consider precisely the strong curvature effects to dynamical symmetry breaking. In fact we will see that going beyond the linear-curvature approximation can lead to qualitatively different results.

In this paper we will investigate some $2 \mathrm{~d}$ and $3 \mathrm{~d}$ four-fermion models which are renormalizable - in the $1 / N$ expansion - in a maximally symmetric constant-curvature space (either of positive or of negative curvature). The renormalized effective potential will be found for any value of the curvature and the possibility of dynamical symmetry breaking in a curved spacetime will be carefully explored. Furthermore, the phase structure of the theory will be described in detail.

The paper is organized as follows. In the next section we calculate the effective potential of composite fermions in the Gross-Neveu model, in the spaces $S^{2}$ and $H^{2}$. The phase diagram in $S^{2}$ is constructed and it is shown that for any value of the coupling constant there exists a curvature above which chiral symmetry is restored. For the case of $H^{2}$, we show that chiral symmetry is always broken. The asymptotic expansions of the effective potential are given explicitly, both for small and for strong curvature. The three-dimensional case 
is studied in Sect. 3. We consider two different four-fermion models: one which exhibits a continuous $\mathrm{U}(2)$ symmetry and another where we concentrate ourselves on two discrete symmetries which happen never to be simultaneously broken (see [16]). In Sect. 4 we study explicitly the dynamical $\mathrm{P}$ and $\mathrm{Z}_{2}$ symmetry breaking pattern in $H^{3}$ and $S^{3}$. Finally, Sect. 5 is devoted to conclusions and some technical points of the procedure are summarized in two appendices.

\section{The Gross-Neveu model in a space of constant cur- vature}

\subsection{Case of the 2d De Sitter space $S^{2}$}

In this subsection we will undertake the discussion of the Gross-Neveu model [2] in De Sitter space. This model, although rather simple in its conception, displays a quite rich structure, similar to that of realistic four-dimensional theories - as renormalizability, asymptotic freedom [2, 17] and dynamical chiral symmetry breaking. Some discussions of chiral symmetry restoration in the Gross-Neveu model for different external conditions (such as an electromagnetic field, non-zero temperature or a change of the fermionic number density) have appeared in the past [18, 21, 30 (the influence of kink-antikink configurations on the phase transitions is described in [22, 23]).

The study of the Gross-Neveu model in an external gravitational field has been performed in Ref. [8] using the Schwinger method [24] (for other analysis of two-dimensional models in curved space, see 25). Unfortunately, the generalization of the Schwinger procedure to curved spacetime is not free from ambiguities and this is why the result of Ref. [8] includes some mistake.

In the calculation of the effective potential in the Gross-Neveu model on De Sitter space we will use a rigorous mathematical treatment of the fermionic propagator in (constant curvature) spacetime, that has been developed in Ref. [26]. We shall start from the action

$$
S=\int d^{2} x \sqrt{-g}\left[\bar{\psi} i \gamma^{\mu}(x) \nabla_{\mu} \psi+\frac{\lambda}{2 N}(\bar{\psi} \psi)^{2}\right],
$$

where $N$ is the number of fermions, $\lambda$ the coupling constant, $\gamma^{\mu}(x)=\gamma^{a} e_{a}^{\mu}(x)$, with $\gamma^{a}$ the ordinary Dirac matrix in flat space, and $\nabla_{\mu}$ is the covariant derivative. By introducing the auxiliary field $\sigma$, it is convenient to rewrite (11) as

$$
S=\int d^{2} x \sqrt{-g}\left[\bar{\psi} i \gamma^{\mu}(x) \nabla_{\mu} \psi-\frac{N}{2 \lambda} \sigma^{2}-\sigma \bar{\psi} \psi\right]
$$

with $\sigma=-\frac{\lambda}{N} \bar{\psi} \psi$. Furthermore, in order to apply the results of Ref. [26], it is convenient to 
use Euclidean notations. Then (2) is written as

$$
S=\int d^{2} x \sqrt{g}\left[\bar{\psi} \gamma^{\mu}(x) \nabla_{\mu} \psi+\frac{N}{2 \lambda} \sigma^{2}+\sigma \bar{\psi} \psi\right] .
$$

Assuming that we work in De Sitter space and using the standard $1 / N$ expansion, we get the effective potential in terms of the $\sigma$ field as follows [8, 10, 11, 9]

$$
V(\sigma)=\frac{\sigma^{2}}{2 \lambda}+\operatorname{Tr} \int_{0}^{\sigma} D(x, x, s) d s,
$$

where the propagator $D$ is defined by

$$
(\widehat{\nabla}+s) D(x, y, s)=-\delta_{2}(x, y) .
$$

The curvature of both $S^{d}$ and $H^{d}$ can be written in the form

$$
R=\frac{d(d-1) k}{a^{2}},
$$

with $k=1$ for $S^{d}$ and $k=-1$ for $H^{d}$; $a$ stands for the radius of the manifold.

We consider first $k=1$. Following [26] we begin the calculation by obtaining the 'squareing' Green's function:

$$
\left(\widehat{\nabla}^{2}-s^{2}\right) G(x, y)=-\delta_{2}(x, y) .
$$

Then $D=(\widehat{\nabla}-s) G(x, y)$. We resort to the Ansatz [26]

$$
G(x, y)=u(x, y) g(p),
$$

where $u(x, y)$ yields a unit matrix in spinor indices when $y \rightarrow x$, and $p$ is the distance between $x$ and $y$ along the geodesic that goes through these two points. Introducing the notations

$$
\theta=\frac{p}{a}, \quad g(\theta)=\cos \frac{\theta}{2} h(\theta), \quad Z=\cos ^{2} \frac{\theta}{2},
$$

this leads to the following equation for $h$

$$
\left[Z(1-Z) \frac{d^{2}}{d Z^{2}}+(2-3 Z) \frac{d}{d Z}-1-s^{2} a^{2}\right] h(\theta)=0 .
$$

One can show [26] that the solution of (7) is given by a linear combination of the hypergeometric functions [27]. As boundary conditions for (7) it is convenient to choose the following. First, one selects the singularity that appears for $\theta \rightarrow 0$. Second, one demands [26] that the singular part of this limit has the same form as in flat space

$$
g(\theta) \sim-\frac{1}{2 \pi} \ln \theta, \quad \theta \rightarrow 0 .
$$

Using properties of the hypergeometric functions and the boundary conditions (111), the function $D$ for coinciding arguments is found to be

$$
D(x, x, s)=-s \lim _{\theta \rightarrow 0} g(\theta)=\frac{s}{4 \pi}\left[\psi(1+i s a)+\psi(1-i s a)+2 \gamma+\ln \frac{\theta^{2}}{4}\right],
$$


where (11) has been used explicitly, $\gamma$ is the Euler constant and $\psi$ the digamma function, and the two arguments of $D$ are supposed to be separated by a small geodesic distance $p=\theta a$. Differentiating (4) with respect to $\sigma$ and using (12), one gets

$$
\begin{aligned}
V^{\prime}(\sigma)= & \frac{\sigma}{\lambda}+\operatorname{Tr} D(x, x, \sigma)=\frac{\sigma}{\lambda}\left\{1+\frac{\lambda}{2 \pi}\left[2 \gamma+\psi\left(1+i|\sigma| \sqrt{\frac{2}{R}}\right)\right.\right. \\
& \left.\left.+\psi\left(1-i|\sigma| \sqrt{\frac{2}{R}}\right)+\ln \frac{p^{2} R}{8}\right]\right\},
\end{aligned}
$$

where $R$ is the curvature.

As renormalization conditions we choose the following [8]

$$
\left.V^{\prime \prime}(\sigma)\right|_{R=0, \sigma=\mu}=\frac{1}{\lambda} .
$$

By selecting the counterterms of the form

$$
\delta V=-\frac{1}{2 \pi} \sigma^{2} \ln \frac{\mu p e^{\gamma+1}}{2},
$$

adding them to (13) and using the asymptotics of $\psi(x)$, we find the following value for the derivative of the renormalized effective potential

$$
V^{\prime}(\sigma)=\frac{\sigma}{\lambda}\left\{1+\frac{\lambda}{2 \pi}\left[\psi\left(1+i|\sigma| \sqrt{\frac{2}{R}}\right)+\psi\left(1-i|\sigma| \sqrt{\frac{2}{R}}\right)-2-\ln \frac{2 \mu^{2}}{R}\right]\right\} .
$$

As we can see, letting aside the different notation employed for $\sigma$, the terms involving $\psi(x)$ differ from the corresponding terms reported in [8].

Starting now from expression (16), different physical questions can be studied. In particular, the possibility to construct a corresponding phase diagram appears. To this end, let us calculate the second derivative

$$
\begin{aligned}
V^{\prime \prime}(\sigma)= & \frac{1}{\lambda}+\frac{1}{2 \pi}\left[\psi\left(1+i|\sigma| \sqrt{\frac{2}{R}}\right)+\psi\left(1-i|\sigma| \sqrt{\frac{2}{R}}\right)-2-\ln \frac{2 \mu^{2}}{R}\right] \\
& +\frac{i|\sigma|}{2 \pi} \sqrt{\frac{2}{R}}\left[\zeta\left(2,1+i|\sigma| \sqrt{\frac{2}{R}}\right)-\zeta\left(2,1-i|\sigma| \sqrt{\frac{2}{R}}\right)\right] .
\end{aligned}
$$

We can now study the behavior of the renormalized $V$ near $\sigma=0$. We have always

$$
V^{\prime}(0)=0, \quad V^{\prime \prime}(0)=\frac{1}{\lambda}\left[1-\frac{\lambda}{2 \pi}\left(2 \gamma+2+\ln \frac{2 \mu^{2}}{R}\right)\right] .
$$

With the notation

$$
R_{0}=2 \mu^{2} e^{2(\gamma+1)}, \quad \lambda_{0}=2 \pi,
$$

we obtain that the point $\sigma=0$ is a minimum for $R>R_{0} \exp \left(-\lambda_{0} / \lambda\right)$ and a maximum for $R<R_{0} \exp \left(-\lambda_{0} / \lambda\right)$. That is, for any value of $\lambda$, there exists a value of the curvature 
above which chiral symmetry is restored. The connection of the critical curvature with the coupling constant is

$$
R_{c r}=R_{0} e^{-\lambda_{0} / \lambda}
$$

a different expression for $R_{c r}$ will be given below, in which the independence of $R_{c r}$ from the renormalization scale $\mu$ is made apparent. For $R<R_{c r}$ the chiral symmetry is broken and a dynamical fermion mass is generated.

As next step one can investigate different limits of the expression (16) for the renormalized potential. For $R \rightarrow 0$, one obtains from (16)

$$
\begin{aligned}
V^{\prime}(\sigma) & \sim \frac{\sigma}{\lambda}\left[1+\frac{\lambda}{2 \pi}\left(\ln \frac{\sigma^{2}}{\mu^{2}}-2+\frac{R}{12 \sigma^{2}}\right)\right], \\
V(\sigma) & \sim \frac{\sigma^{2}}{2 \lambda}\left[1+\frac{\lambda}{2 \pi}\left(\ln \frac{\sigma^{2}}{\mu^{2}}-3\right)\right]+\frac{R}{48 \pi} \ln \frac{\sigma^{2}}{R} .
\end{aligned}
$$

Notice that in (22) the constant of integration has been chosen having in mind the finiteness of $V$ as $R \rightarrow 0$ [8]. At the same time, at $R \rightarrow \infty$ one gets

$$
\begin{aligned}
V^{\prime}(\sigma) & \sim \frac{\sigma}{\lambda}\left[1+\frac{\lambda}{2 \pi}\left(-2 \gamma-2+4 \zeta(3) \frac{\sigma^{2}}{R}-\ln \frac{2 \mu^{2}}{R}\right)\right], \\
V(\sigma) & \sim \frac{\sigma^{2}}{2 \lambda}\left[1+\frac{\lambda}{2 \pi}\left(-2 \gamma-2+2 \zeta(3) \frac{\sigma^{2}}{R}-\ln \frac{2 \mu^{2}}{R}\right)\right] .
\end{aligned}
$$

The last expressions show the behavior of the effective potential at strong curvature. Analysing (21)-(24) one can see that for small $R$ chiral symmetry is broken, as it happens in flat spacetime. However, in the limit of strong curvature chiral symmetry is restored. Thus, the study of the asymptotics is a further check of our general analysis (phase diagrams).

If one defines $M$ by

$$
\left.V^{\prime \prime}(\sigma)\right|_{R=0, \sigma=M}=0,
$$

then one can express the derivative of the effective potential in terms of this parameter, as

$$
V^{\prime}(\sigma)=\frac{\sigma}{2 \pi}\left[\psi\left(1+i \sqrt{\frac{2 \sigma^{2}}{R}}\right)+\psi\left(1-i \sqrt{\frac{2 \sigma^{2}}{R}}\right)+\ln \frac{R}{2 M^{2}}\right] .
$$

We may now rephrase the criterion of symmetry restoration in terms of $M$ by saying that the symmetry is restored when $R>R_{c r}=2 M^{2} \exp (2 \gamma)$. The shape of the effective potential

for different values of the quotient $\frac{R}{M^{2}}$ is shown in Fig. 1. The character of the transition is continuous, as illustrated by Fig. 2 .

\subsection{Case of the hyperbolic space $H^{2}$}

In the hyperbolic space $H^{2}$ (negative curvature) the analysis can be carried out in a very similar way. After introducing the notations

$$
\theta=\frac{p}{a}, \quad g(\theta)=\cosh \frac{\theta}{2} h(\theta), \quad Z=\cosh ^{2} \frac{\theta}{2},
$$


Eq. (7) acquires the form [26]

$$
\left[Z(1-Z) \frac{d^{2}}{d Z^{2}}+(2-3 Z) \frac{d}{d Z}-1+s^{2} a^{2}\right] h(\theta)=0 .
$$

As a result, using a similar procedure as in Sect. 2.1, we get

$$
D(x, x, s)=\frac{s}{4 \pi}\left[2 \gamma+\psi(1+s a)+\psi(s a)+\ln \frac{\theta^{2}}{4}\right] .
$$

The non-renormalized expression for $V^{\prime}(\sigma)$ is

$$
V^{\prime}(\sigma)=\frac{\sigma}{\lambda}\left\{1+\frac{\lambda}{2 \pi}\left[2 \gamma+\psi\left(1+|\sigma| \sqrt{\frac{2}{|R|}}\right)+\psi\left(|\sigma| \sqrt{\frac{2}{|R|}}\right)+\ln \frac{p^{2}|R|}{8}\right]\right\} .
$$

Making the same renormalization as in Sect. 2.1, we obtain the renormalized effective potential

$$
\begin{aligned}
V^{\prime}(\sigma)= & \frac{\sigma}{\lambda}\left\{1+\frac{\lambda}{2 \pi}\left[2 \psi\left(1+|\sigma| \sqrt{\frac{2}{|R|}}\right)-2-\ln \frac{2 \mu^{2}}{|R|}\right]\right\}-\sqrt{\frac{|R|}{8 \pi^{2}}} \\
V^{\prime \prime}(\sigma)= & \frac{1}{\lambda}\left\{1+\frac{\lambda}{2 \pi}\left[2 \psi\left(1+|\sigma| \sqrt{\frac{2}{|R|}}\right)-2-\ln \frac{2 \mu^{2}}{|R|}\right]\right\} \\
& +\frac{|\sigma|}{\pi} \sqrt{\frac{2}{|R|}} \zeta\left(2,1+|\sigma| \sqrt{\frac{2}{|R|}}\right) .
\end{aligned}
$$

A careful study of $V^{\prime}(0)$ shows that due to the presence of the last term in (29), $\sigma=0$ is never stationary for any value of $\lambda$ and finite $R$. Owing to the fact that $V^{\prime}(0)<0$, chiral symmetry is always broken in $H^{2}$.

In the small curvature limit $(|R| \rightarrow 0)$

$$
V(\sigma)=\frac{\sigma^{2}}{2 \lambda}\left[1+\frac{\lambda}{2 \pi}\left(\ln \frac{\sigma^{2}}{\mu^{2}}-3\right)\right]-\frac{|R|}{48 \pi} \ln \frac{\sigma^{2}}{|R|},
$$

what coincides with (22), taking into account the change of sign for the curvature. In the strong curvature limit $(|R| \rightarrow \infty)$

$$
V(\sigma)=\frac{\sigma^{2}}{2 \lambda}\left[1-\frac{\lambda}{2 \pi}\left(2 \gamma+2+\ln \frac{2 \mu^{2}}{|R|}+\frac{\sqrt{2|R|}}{|\sigma|}\right)\right] .
$$

The analysis of Eqs. (31) and (32) shows that the general conclusion about the chiral symmetry breaking at any finite $R$ in $H^{2}$ is correct. In a similar way one can study the influence of curvature in the massive Gross-Neveu model (for a recent discussion of such model at non-zero temperature, see [29]). 


\section{Dynamical U(2) flavor symmetry breaking in $H^{3}$ and $S^{3}$}

This section is devoted to the description of a three-dimensional four-fermion model which has a continuous flavor-like symmetry and how its breaking is affected by a gravitational background.

We consider the following model on a Riemannian manifold

$$
\mathcal{L}_{E}=\bar{\psi} \not D \psi-\frac{\lambda_{B}}{2 N}\left[(\bar{\psi} \psi)^{2}+\left(\bar{\psi} i \tau^{1} \psi\right)^{2}+\left(\bar{\psi} i \gamma^{5} \psi\right)^{2}\right]
$$

with

$\gamma^{\mu}=\left(\begin{array}{cc}\sigma^{\mu} & 0 \\ 0 & -\sigma^{\mu}\end{array}\right), \quad \mu=1,2,3, \quad \gamma^{5}=i\left(\begin{array}{cc}0 & \mathbb{1}_{2} \\ -\mathbb{1}_{2} & 0\end{array}\right)=-\tau^{1} \gamma^{1} \gamma^{2} \gamma^{3}, \quad \tau^{1}=\left(\begin{array}{cc}0 & \mathbb{1}_{2} \\ \mathbb{1}_{2} & 0\end{array}\right)$,

so we take a reducible, four-dimensional Dirac algebra.

The transformation of the bilinear terms $\bar{\psi} \psi, \bar{\psi} i \tau^{1} \psi$ and $\bar{\psi} i \gamma^{5} \psi$ under

$$
\delta \psi=-i T_{\alpha} \theta^{\alpha} \psi, \quad \delta \bar{\psi}=i \bar{\psi} \gamma^{3} T_{\alpha} \gamma^{3} \theta^{\alpha}
$$

with $T_{0}=1, T_{1}=\gamma^{5}, T_{2}=\tau^{1}$ and $T_{3}=i \tau^{1} \gamma^{5}$, is given by

$$
\left(\begin{array}{c}
\delta(\bar{\psi} \psi) \\
\delta\left(\bar{\psi} i \tau^{1} \psi\right) \\
\delta\left(\bar{\psi} i \gamma^{5} \psi\right)
\end{array}\right)=2\left(\begin{array}{ccc}
0 & -\theta^{1} & -\theta^{2} \\
\theta^{1} & 0 & -\theta^{3} \\
\theta^{2} & \theta^{3} & 0
\end{array}\right)\left(\begin{array}{c}
\bar{\psi} \psi \\
\bar{\psi} i \tau^{1} \psi \\
\bar{\psi} i \gamma^{5} \psi
\end{array}\right) .
$$

It is convenient to express this theory in terms of auxiliary fields, namely

$$
\mathcal{L}_{E}=\bar{\psi}\left(\not D+\sigma+i \tau^{1} \rho+i \gamma^{5} \pi\right) \psi+\frac{N}{2 \lambda_{B}}\left(\sigma^{2}+\rho^{2}+\pi^{2}\right)
$$

what yields

$$
\Gamma_{N \rightarrow \infty}[\sigma, \rho, \pi]=\int d x \sqrt{g} \frac{N}{2 \lambda_{B}}\left(\sigma^{2}+\rho^{2}+\pi^{2}\right)-N \ln \operatorname{det}\left(\not D+\sigma+i \tau^{1} \rho+i \gamma^{5} \pi\right) .
$$

We consider the constant configurations of $\sigma, \rho, \pi$. The rotational symetries impose that the effective potential only depends on $\sigma^{2}+\rho^{2}+\pi^{2}$, what allows us to set $\rho=\pi=0$. The regularized expression of the effective potential turns out to be:

$$
\int d x \sqrt{g} V[\sigma]=\int d x \sqrt{g} \frac{N}{2 \lambda_{B}} \sigma^{2}+\frac{N}{2} \operatorname{Tr} \int_{1 / \Lambda^{2}}^{\infty} \frac{d t}{t} e^{-t \sigma^{2}} e^{\nabla^{2} t} .
$$

The reader may verify that the use of point-splitting regularization leads to the same results that we get below by regulating the lower limit in the proper time integral. On the right 
hand side we observe the appearance of the coincidence limit of the heat kernel. Resorting to the results of appendix $\mathrm{B}$, the outcome is (for $H^{3}$ )

$$
V[\sigma]=\frac{N}{2 \lambda_{B}} \sigma^{2}+\frac{N}{2 \pi^{3 / 2}} \int_{1 / \Lambda^{2}}^{\infty} \frac{d t}{t^{5 / 2}}\left(1+\frac{1}{2 t}\right) e^{-t \sigma^{2}} e^{\nabla^{2} t} .
$$

The dependence on the radius of the manifold is not shown, but it can very easily be recovered from dimensional analysis. We obtain

$$
\frac{1}{N} V[\sigma]=\frac{\sigma^{2}}{2 \lambda_{B}}+\frac{|\sigma|}{(4 \pi)^{3 / 2} a^{2}}\left[\Gamma\left(-\frac{1}{2}, \frac{\sigma^{2}}{\Lambda^{2}}\right)+2 a^{2} \sigma^{2} \Gamma\left(-\frac{3}{2}, \frac{\sigma^{2}}{\Lambda^{2}}\right)\right] .
$$

Imposing the renormalization condition

$$
\frac{1}{N} \frac{d^{2}}{d \sigma^{2}} V\left[\sigma=0, R=-6 / a^{2}=0\right]=\frac{1}{\lambda},
$$

one gets

$$
\frac{1}{\lambda_{B}}=\frac{1}{\lambda}+\frac{\Lambda}{\pi^{3 / 2}}
$$

and in the limit $\Lambda \rightarrow \infty$ :

$$
\frac{1}{N} V[\sigma]=\frac{\sigma^{2}}{2 \lambda}+\frac{|\sigma|}{\pi}\left(\frac{\sigma^{2}}{3}-\frac{1}{4 a^{2}}\right)
$$

From this one can easily see that the symmetry is always broken in $H^{3}$. In fact we can also notice the curious feature that the origin is not a solution of the gap equation. This appears to liken the situation of four-fermion models in three dimensions under the influence of a magnetic field (see [28]).

As for $S^{3}$, we have (see appendix B).

$$
V(\sigma)=\frac{\sigma^{2}}{2 \lambda_{B}}-\frac{1}{2 V o l} \operatorname{Tr} \int_{0}^{\sigma^{2}} d s \frac{1}{s-\not \partial^{2}} .
$$

From this formula one derives the regularized effective potential using the result from 26] that on $S^{N}$ the solution of

$$
\left(\not{ }^{2}-m^{2}\right) G(y)=-\delta_{N}(y)
$$

is given by $G(y)=U(y) g_{N}(\sigma)$, where $\mathrm{U}(\mathrm{y})$ is a parallel transport matrix that is the identity at the coincidence limit, and

$$
g_{N}(\theta)=\frac{\Gamma\left(\frac{N}{2}+i m\right) \Gamma\left(\frac{N}{2}-i m\right)}{(4 \pi)^{N / 2} \Gamma\left(\frac{N}{2}+1\right)} \cos \left(\frac{\theta}{2}\right) F\left(\frac{N}{2}+i m, \frac{N}{2}-i m, \frac{N}{2}+1, \cos ^{2}\left(\frac{\theta}{2}\right)\right)
$$

where $\theta=\frac{\rho}{a}$, ( $\rho$ is the geodesic distance, and a is the radius of the manifold). With this in mind and using known properties of the hypergeometric functions one may arrive at the 
following expression (in which we only keep terms divergent in $\rho$ and terms independent from it)

$$
V^{\prime}(\sigma)=\frac{\sigma}{\lambda_{B}}-\frac{\sigma}{4 \pi} \frac{\operatorname{Tr} \mathbb{1}_{4}}{\rho}+\frac{\operatorname{Tr} \mathbb{1}_{4}}{4 \pi}\left(\frac{1}{4 a^{2}}+\sigma^{2}\right) \tanh (\pi \sigma a)
$$

Using the renormalization given by

$$
\frac{1}{N} \frac{d^{2}}{d \sigma^{2}} V\left[\sigma=0, R=6 / a^{2}=0\right]=\frac{1}{\lambda}
$$

we get

$$
V^{\prime}(\sigma)=\frac{\sigma}{\lambda}+\frac{1}{\pi}\left(\frac{1}{4 a^{2}}+\sigma^{2}\right) \tanh (\pi \sigma a)
$$

This appears to be in agreement with the correspondent effective potential in the GrossNeveu model (in the simplest version without continuous $U(2)$ symmetry and discrete symmetries) on De Sitter space using, different from our approach, dimensional regularization (see [31]). We can now compare this result with the one that was found in Ref. [9], which was a study of three-dimensional theories in the small curvature limit. One can easily check that expression (46) is indeed compatible with those in [9]. What is most surprising in the weak curvature limit is that, in view of the results, one might be tempted to conclude that the origin is not a solution to the gap equation (as it happens in $H^{3}$ ), and, furthermore, that there may be no solution at all. However, looking at the exact result (46) or (47), this is seen to be an artifact of the approximation. The effective potential given in expression (47) may give rise to a second order phase transition, as we illustrate in Fig 3.

\section{Dynamical $\mathbf{P}$ and $\mathbf{Z}_{2}$ symmetry breaking in $H^{3}$ and $S^{3}$}

In this section we analyse a model which displays two discrete symmetries. First of all, we present the model and later we describe its symmetries in some detail. To finish, we will describe the influence of gravity on the breaking of the symmetries. Using the representation for the $\gamma^{\mu}$ (which has no $\gamma_{5}$ )

$$
\gamma^{\mu}=\left(\begin{array}{cc}
\sigma^{\mu} & 0 \\
0 & \sigma^{\mu}
\end{array}\right)
$$

it is also possible in this case to define a parity operation which admits the presence of a mass term, i.e.

$$
\mathcal{L}_{E}=\bar{\psi} \not \partial \psi+m \bar{\psi} \tau^{3} \psi
$$

with

$$
\left.P\left[\psi\left(x_{1}, x_{2}, x_{3}\right)\right]=\gamma^{2} \tau^{1} \psi\left(x_{1},-x_{2}, x_{3}\right), \quad P\left[\bar{\psi}\left(x_{1}, x_{2}, x_{3}\right)\right]=-\bar{\psi}\left(x_{1},-x_{2}, x_{3}\right) \gamma^{1} \tau^{1}\right]
$$


However, $\bar{\psi} \psi$ transforms as a pseudoscalar. There is another discrete symmetry of the kinetic term, given by

$$
Z_{2}[\psi]=\tau^{1} \psi, \quad Z_{2}[\bar{\psi}]=\bar{\psi} \tau^{1}
$$

Under this operation, the roles of the mass terms are reversed, e.g.

$$
Z_{2}\left[\bar{\psi} \tau^{3} \psi\right]=-\bar{\psi} \tau^{3} \psi, \quad Z_{2}[\bar{\psi} \psi]=\bar{\psi} \psi
$$

With this in mind, it is immediate that

$$
\mathcal{L}_{E}=\bar{\psi} \not D \psi-\frac{\lambda_{B}}{2 N}(\bar{\psi} \psi)^{2}-\frac{\kappa_{B}}{2 N}\left(\bar{\psi} \tau^{3} \psi\right)^{2}
$$

is invariant under both $\mathrm{P}$ and $\mathrm{Z}_{2}$. But, as $\bar{\psi} \tau^{3} \psi$ is not invariant under $\mathrm{Z}_{2}$, it can be taken as an order parameter for the $\mathrm{Z}_{2}$ symmetry breaking. Likewise, $\langle\bar{\psi} \psi\rangle$ is an order parameter for the $\mathrm{P}$ symmetry breaking.

In terms of auxiliary fields

$$
\mathcal{L}_{E}=\bar{\psi}\left(\not D+\phi+\chi \tau^{3}\right) \psi+\frac{N}{2 \lambda_{B}} \phi^{2}+\frac{N}{2 \kappa_{B}} \chi^{2} .
$$

Proceeding along the same lines we have trodden before, we will impose equivalent renormalization conditions

$$
\frac{d^{2}}{d \phi^{2}} V[\phi=0, R=0]=\frac{1}{\lambda}, \quad \frac{d^{2}}{d \chi^{2}} V[\chi=0, R=0]=\frac{1}{\kappa} .
$$

In the case of hyperbolic space $H^{3}$ we get that the effective potential in the large- $N$ limit is given by

$$
\begin{aligned}
\frac{1}{N} V[\phi, \chi]= & \frac{\sigma_{+}^{2}+\sigma_{-}^{2}}{8}\left(\frac{1}{\lambda}+\frac{1}{\kappa}\right)+\frac{\sigma_{+} \sigma_{-}}{4}\left(\frac{1}{\lambda}-\frac{1}{\kappa}\right) \\
& +\frac{1}{2 \pi}\left[\left|\sigma_{+}\right|\left(\frac{\sigma_{+}^{2}}{3}-\frac{1}{4 a^{2}}\right)+\left|\sigma_{-}\right|\left(\frac{\sigma_{-}^{2}}{3}-\frac{1}{4 a^{2}}\right)\right],
\end{aligned}
$$

with $\sigma_{ \pm} \equiv \phi \pm \chi$. We obtain the two cases

$$
\frac{1}{\lambda}-\frac{1}{\kappa}>0, \quad \bar{\sigma}_{+}=-\bar{\sigma}_{-}, \quad(\bar{\phi}=0, \bar{\chi} \neq 0)
$$

for the $\mathrm{Z}_{2}$ symmetry breaking, and

$$
\frac{1}{\lambda}-\frac{1}{\kappa}<0, \quad \bar{\sigma}_{+}=\bar{\sigma}_{-}, \quad(\bar{\phi} \neq 0, \bar{\chi}=0),
$$

for the $\mathrm{P}$ symmetry breaking, respectively. This is illustrated in Figs. 4 and 5, where we only consider positive values of $\phi$, as the symmetry of the model allows us to reproduce the result for the region of negative $\phi$ immediately. It is also worth noting that either $\mathrm{P}$ or $\mathrm{Z}_{2}$ is broken, but that it is impossible to have both symmetries broken. Fig. 4 exemplifies the 
first situation (2.68): one sees that the minimum lies at $\phi=0, \chi \neq 0$ and that $\mathrm{Z}_{2}$ is broken. In Fig. 5 we are in the second situation: the minimum lies at $\phi \neq 0, \chi=0$ and $\mathrm{P}$ is broken.

As for the $S^{3}$ case, the reader may verify - along the same lines as above - that one encounters again two different situations: either both $P$ and $Z_{2}$ are unbroken or just one of them is broken. This is obtained without difficulty by repeating the same analysis, and we feel that further details are not necessary.

\section{Conclusions}

In this paper we have reviewed a powerful method for the exact study of spinor heat kernels and propagators on maximally symmetric spaces. We have used it with success in a number of different four-fermion models. The renormalized effective potential has been found, in each case, for any value of the curvature, and its asymptotic expansion has been given explicitly, both for small and for strong curvature.

We have described in great detail the influence of gravity on the dynamical symmetry breaking pattern of some $\mathrm{U}(2)$ flavor-like and discrete symmetries. In particular, we have seen explicitly that the effect of a negative curvature is similar to that of the presence of a magnetic field.

In the case of positive curvature, $S^{3}$, we have checked that the scenario given in the framework of a small curvature expansion is dramatically changed when we treat gravity exactly. In particular we find that the character of the phase transitions induced by gravity is continuous and that the origin is always a solution of the gap equation. A point to be duely remarked is the fact that the techniques applied here to two- and three-dimensional models work equally well in four- and higher-dimensional ones, as well. Of course, these models are not renormalizable in the standard way (see however, refs. [32] where mean field renormalization of four-fermion models has been discussed), but one can still apply the above method in order to obtain the cut-off dependent effective potential, what can certainly be useful for cosmological applications.

Another quite interesting topic is to study a formulation overlapping technique between the Schwinger-Dyson equations in a constant-curvature space and the effective potential approach. We hope to address some of these questions in the near future.

\section{Acknowledgments}

SL gratefully acknowledges an FPI grant from Generalitat de Catalunya. YIS is indebted with Dr. A. Letwin for kind support. This work has been partially financed by DGICYT (Spain), projects PB93-0035 and SAB93-0024, and by RFFR (Russia), project 94-02-03234. 


\section{A Appendix: Generalities about spinors in three di- mensions}

Some general facts about spinors in three dimensions that may be interesting to recall for the benefit of the reader are the following. The Dirac algebra $\left\{\gamma_{\mu}, \gamma_{\nu}\right\}=2 g_{\mu \nu}$ has two irreducible representations, namely

1. $\gamma^{0}=\sigma^{3}, \gamma^{1}=i \sigma^{1}, \gamma^{2}=i \sigma^{2}$

and

2. $\gamma_{\mu}^{\prime}=-\gamma_{\mu}$.

On the other hand, there is no ' $\gamma^{5}$ ' matrix in order 2 which anticommutes with all of the $\gamma^{\mu}$ s. A mass term of the form $m \bar{\psi} \psi$ in the Lagrangian explicitly violates parity, defined by

$$
P: \psi\left(x^{0}, x^{1}, x^{2}\right) \longrightarrow \sigma^{1} \psi\left(x^{0},-x^{1}, x^{2}\right)
$$

However, if one uses a reducible representation of the Dirac matrices, such as

$$
\gamma^{0}=\left(\begin{array}{cc}
\sigma^{3} & 0 \\
0 & -\sigma^{3}
\end{array}\right), \quad \gamma^{1}=\left(\begin{array}{cc}
i \sigma^{1} & 0 \\
0 & -i \sigma^{1}
\end{array}\right), \quad \gamma^{2}=\left(\begin{array}{cc}
i \sigma^{2} & 0 \\
0 & -i \sigma^{2}
\end{array}\right)
$$

then the mass term $m \bar{\psi} \psi$ does preserve parity, as defined by

$$
P: \psi\left(x^{0}, x^{1}, x^{2}\right) \longrightarrow \tau^{1} \gamma^{1} \psi\left(x^{0},-x^{1}, x^{2}\right)
$$

being

$$
\tau^{1}=\left(\begin{array}{cc}
0 & \mathbb{1}_{2} \\
\mathbb{1}_{2} & 0
\end{array}\right), \quad \tau^{2}=\left(\begin{array}{cc}
0 & -i \mathbb{1}_{2} \\
i \mathbb{1}_{2} & 0
\end{array}\right), \quad \tau^{3}=\left(\begin{array}{cc}
\mathbb{1}_{2} & 0 \\
0 & -\mathbb{1}_{2}
\end{array}\right) .
$$

The kinetic term is invariant under the $\mathrm{U}(2)$ transformations with generators $T_{0}=$ $\mathbb{1}_{4}, T_{1}=\gamma^{5}, T_{2}=\tau^{1}$, and $T_{3}=i \tau^{1} \gamma^{5}$, where

$$
\gamma^{5}=\tau^{1} \gamma^{0} \gamma^{1} \gamma^{2}=i\left(\begin{array}{cc}
0 & \mathbb{1}_{2} \\
-\mathbb{1}_{2} & 0
\end{array}\right)
$$

The mass term breaks this symmetry down to $\mathrm{U}(1) \times \mathrm{U}(1)$ with the generators $T_{0}, T_{3}$.

\section{B Appendix: Spinor heat-kernel in maximally sym- metric spaces (the intertwining method)}

In this appendix we review a powerful method to derive closed expressions for the spinor heat-kernel and propagators in maximally symmetric spaces (for more details see [26]). 
The spinor heat kernel satisfies $\left(-\frac{\partial}{\partial t}+\not^{2}\right) K\left(y, y_{0} ; t\right)=0$ and the initial condition $\lim _{t \rightarrow 0}$ $K\left(y, y_{0} ; t\right)=1 \delta_{N}\left(y, y_{0}\right)$. Substituting the Ansatz $K(y, t)=U(y) f(\sigma, t)$ in the heat-kernel equation, one gets

$$
-U \frac{\partial f}{\partial t}+U \nabla^{a} \nabla_{a} f+2 n^{a}\left(\nabla_{a} U\right) \frac{\partial f}{\partial \sigma}+\left(\nabla^{a} \nabla_{a} U\right) f-\frac{R}{4} U f=0,
$$

where $n_{a}=\nabla_{a} \sigma$. Here the term linear in $\partial f / \partial \sigma$ and $\nabla_{a} U$ cancels out, provided that $U$ satisfies the parallel transport equation

$$
n^{a} \nabla_{a} U=0, \quad U\left(y_{0}\right)=1 .
$$

The Laplacian acting on $f$ can be replaced by its radial part given by

$$
\nabla^{a} \nabla_{a} f=\square_{N} f=\left(\partial_{\sigma}^{2}+(N-1) B \partial_{\sigma}\right) f, \quad B= \begin{cases}\frac{1}{a} \cot \left(\frac{\sigma}{a}\right), & S^{N}, \\ \frac{1}{a} \operatorname{coth}\left(\frac{\sigma}{a}\right), & H^{N},\end{cases}
$$

and the Laplacian acting on $U$ is

$$
\nabla^{a} \nabla_{a} U=-\frac{1}{4} A^{2}(N-1) U, \quad A=\left\{\begin{array}{cc}
-\frac{1}{a} \tan \left(\frac{\sigma}{2 a}\right), & S^{N}, \\
\frac{1}{a} \tanh \left(\frac{\sigma}{2 a}\right), & H^{N},
\end{array}\right.
$$

The equation for the scalar $f$ becomes

$$
\left(-\partial_{t}+L_{N}\right) f=0, \quad L_{N}=\square_{N}-\frac{R}{4}-\frac{1}{4}(N-1) A^{2} .
$$

The idea is now to relate the solutions of this equation for different $N$ s. To this end one looks for an operator $D$ such that $L_{N} D=D L_{N-2}$. A simple Ansatz leads to

$$
D= \begin{cases}\frac{1}{2 \pi} \cos \frac{\theta}{2} \frac{\partial}{\partial(\cos \theta)}\left(\cos \frac{\theta}{2}\right)^{-1}, & \theta=\frac{\sigma}{a}, \quad S^{N} \\ -\frac{1}{2 \pi} \cosh \frac{x}{2} \frac{\partial}{\partial(\cosh x)}\left(\cosh \frac{x}{2}\right)^{-1}, & x=i \frac{\sigma}{a}, \quad H^{N}\end{cases}
$$

The odd-dimensional case is elementary, taking into account that $L_{N} D^{(N-1) / 2}=D^{(N-1) / 2} L_{1}$ and the known solution of $\left(-\partial_{t}+L_{1}\right) K_{1}=0$. This yields

$$
\begin{gathered}
K_{N}(y, t)=U(y) \cosh \frac{x}{2}\left(-\frac{1}{2 \pi} \frac{\partial}{\partial(\cosh x)}\right)^{(N-1) / 2}\left(\cosh \frac{x}{2}\right)^{-1} \frac{e^{-x^{2} /(4 t)}}{\sqrt{4 \pi t}}, H^{N} \\
K_{N}(y, t)=U(y) \sum_{n=-\infty}^{\infty}(-1)^{n} f_{d p}(\theta+2 \pi n, t), \\
f_{d p}(\theta+2 \pi n, t)=\cosh \frac{\theta}{2}\left(\frac{1}{2 \pi} \frac{\partial}{\partial(\cosh \theta)}\right)^{(N-1) / 2}\left(\cosh \frac{\theta}{2}\right)^{-1} \frac{e^{-\theta^{2} /(4 t)}}{\sqrt{4 \pi t}}, \quad S^{N} .
\end{gathered}
$$

In the particular case $N=3$ these expressions read — in the coincidence limit - as follows

$$
\begin{gathered}
\lim _{y \rightarrow y_{0}} K(y, t)=\mathbb{1}_{d} \frac{1}{(4 \pi t)^{3 / 2}}\left[1+\frac{t}{2 a^{2}}\right], \quad H^{3}, \\
\lim _{y \rightarrow y_{0}} K(y, t)=\mathbb{1}_{d} \frac{1}{(4 \pi t)^{3 / 2}}\left[1-\frac{t}{2 a^{2}}+\sum_{n=1}^{\infty}(-1)^{n} \exp \left(-\frac{\pi^{2} a^{2} n^{2}}{t}\right)\left(2-t-4 \pi^{2} n^{2} \frac{a^{2}}{t}\right)\right], \quad S^{3},
\end{gathered}
$$

where $d$ is the dimension of the Dirac algebra, in our case $d=4$. 


\section{References}

[1] Y. Nambu and G. Jona-Lasinio, Phys. Rev. 122 (1961) 345.

[2] D.J. Gross and A. Neveu, Phys. Rev. D10 (1974) 3235.

[3] B. Rosenstein, B.J. Warr and S.M. Park, Phys. Rep. 205 (1991) 59.

[4] Y. Nambu, in Proc. Int. Workshop on New Trends in Strong Coupling Gauge Theories, Nagoya, 1988; W. Marciano, Phys. Rev. Lett. 62 (1989) 2723; K. Yamawaki, in Proc. 1990 Int. Workshop on Strong Coupling Gauge Theories (World Sci., Singapore, 1991).

[5] W.A. Bardeen, C.T. Hill and M. Lindner, Phys. Rev. D41 (1990) 1647.

[6] A. Hasenfratz, P. Hasenfratz, K. Jansen, J. Kuti and Y. Shen, Nucl. Phys. B365 (1991) 73.

[7] S. Khlebnikov and R.D. Peccei, Phys. Rev. D48 (1993) 361.

[8] I.L. Buchbinder and E.N. Kirillova, Int. J. Mod. Phys A4 (1989) 143.

[9] E. Elizalde, S.D. Odintsov and Yu.I. Shil'nov, Mod. Phys. Lett. A9 (1994) 931.

[10] T. Inagaki, T. Muta and S.D. Odintsov, Mod. Phys. Lett. A8 (1993) 2117.

[11] E. Elizalde, S. Leseduarte and S.D. Odintsov, Phys. Rev. D49 (1994) 5551.

[12] E. Elizalde, S. Leseduarte and S.D. Odintsov, Phys. Lett. B347 (1995) 33.

[13] C.T. Hill and D.S. Salopek, Ann. Phys. (NY) 213 (1992) 21; T. Muta and S.D. Odintsov, Mod. Phys. Lett. A6 (1991) 3641.

[14] E. Elizalde and S.D. Odintsov, Phys. Rev. D (1995), to appear.

[15] I.L. Buchbinder, S.D. Odintsov and I.L. Shapiro, Effective action in quantum gravity (IOP, Bristol and Philadelphia, 1992).

[16] G. W. Semenoff and L. C. R. Wijewardhana, Phys. Rev. Lett. 63 (1989) 2633.

[17] K.G. Wilson, Phys. Rev. D7 (1973) 2911.

[18] B.J. Harrington and A. Yildiz, Phys. Rev. D11 (1975) 1499.

[19] R. Dashen, S. Ma and R. Rajaraman, Phys. Rev. D11 (1975) 775; R. Dashen, B. Hasslacher and A. Neveu, Phys. Rev. D12 (1975) 2443.

[20] L. Jacobs, Phys. Rev. D10 (1974) 3959. 
[21] W. Dittrich and B.G. Englert, Nucl. Phys. B179 (1981) 85.

[22] F. Karsch, J. Kogut and H.W. Wyld, Nucl. Phys. B280 (1987) 289.

[23] M. Mitchard, A.C. Davis and A.J. Macfarlane, Nucl. Phys. B325 (1989) 470.

[24] J. Schwinger, Phys. Rev. 82 (1951) 664.

[25] I. Sachs and A. Wipf, Phys. Lett. B326 (1994) 105.

[26] R. Camporesi, Commun. Math. Phys. 148 (1992) 283.

[27] A. Érdelyi et al., Higher Transcendental Functions (1953), vol. 2.

[28] I. V. Krive and S. A. Naftulin, Phys. Rev. D46 (1992) 2737.

[29] A. Barducci, R. Casalbuoni, M. Modugno, G. Pettini and R. Gatto, Phys. Rev. D51 (1995) 3042.

[30] E. Witten, Nucl. Phys. B145 (1978) 110.

[31] T. Inagaki, S. Mukaigawa and T. Muta, hep-th/9505058.

[32] F. Cooper, G. Guralnik and N. J. Snyderman, Phys. Rev. Lett, 40 (1978) 1620; G. Guralnik and K. Tamvakis, Phys. Rev. D18 (1978) 4551. 


\section{Figure captions}

Fig. 1. In this figure, $v \equiv V a^{2}, s \equiv \sigma a$ and $r \equiv \frac{R}{M^{2}}$. One sees that for $r>r c \equiv \frac{R_{c r}}{M^{2}}$ the symmetry is restored in $S^{2}$.

Fig. 2. In this figure, $r \equiv \frac{R}{M^{2}}$ and $\min =a \sigma_{\min }$, where $\sigma_{\min }$ is the value of $\sigma$ at which $V$ attains its minimum. The continuous character of the transition which takes place in $S^{2}$ is clearly exhibited.

Fig. 3. Here, $v \equiv V a^{3}, s \equiv \sigma a$ and $g \equiv \frac{-\lambda}{a}$. It is clearly seen that there may be a second order phase transition in $S^{3}$ and that it takes place when $g=4$.

Fig. 4. This figure is a plot of $V a^{3}$ in $H^{3}$ in terms of $s \equiv \phi a$ and $t \equiv \chi a$. We have taken $\frac{a}{\lambda}=1$ and $\frac{a}{\kappa}=0.25$, so $\frac{1}{\lambda}-\frac{1}{\kappa}>0$ and $\mathrm{Z}_{2}$ is broken.

Fig. 5. This is also a plot of $V a^{3}$ in $H^{3}$, with $s$ and $t$ defined as in Fig. 4. Here we have $\frac{1}{\lambda}-\frac{1}{\kappa}<0$ and $\mathrm{P}$ is broken. 


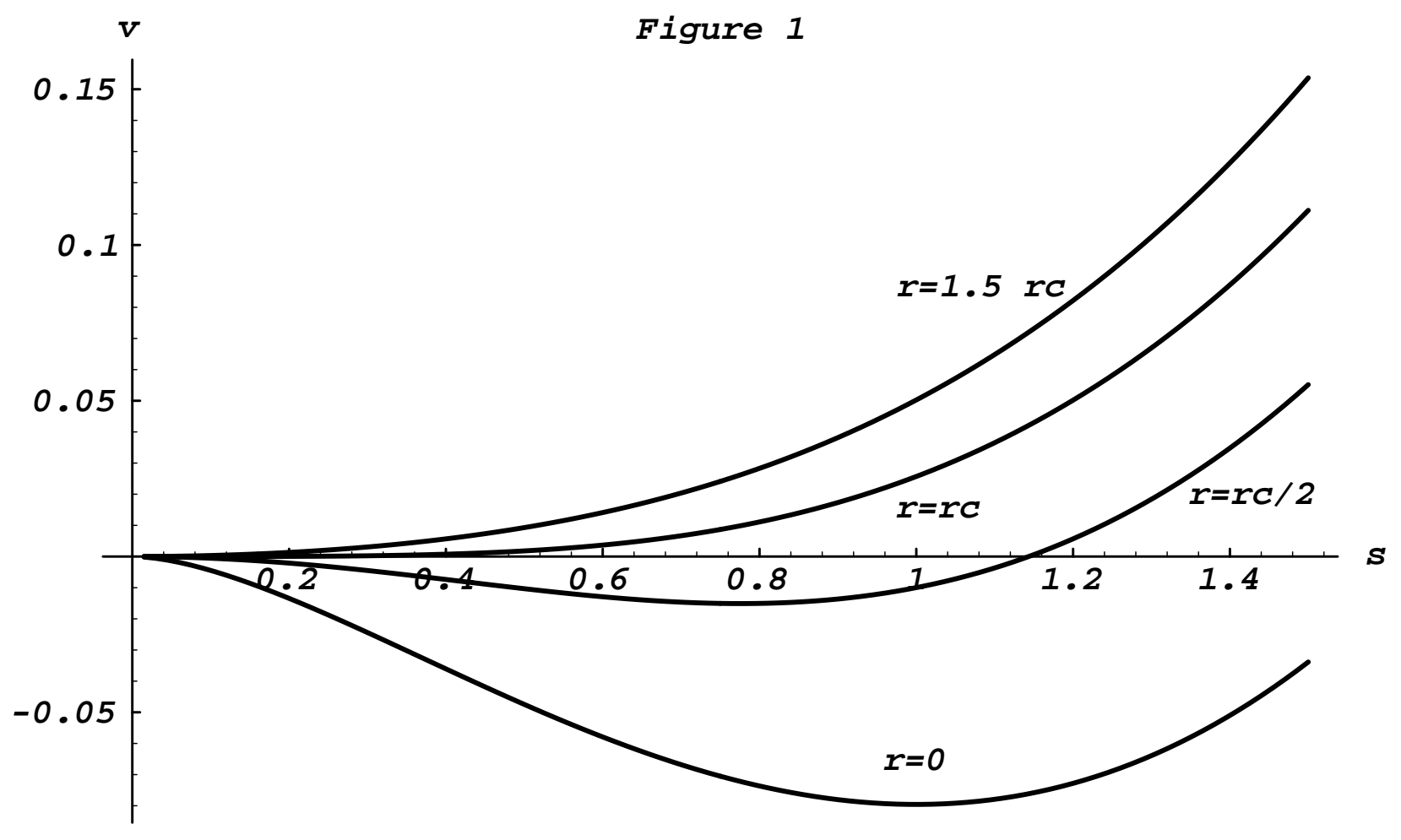




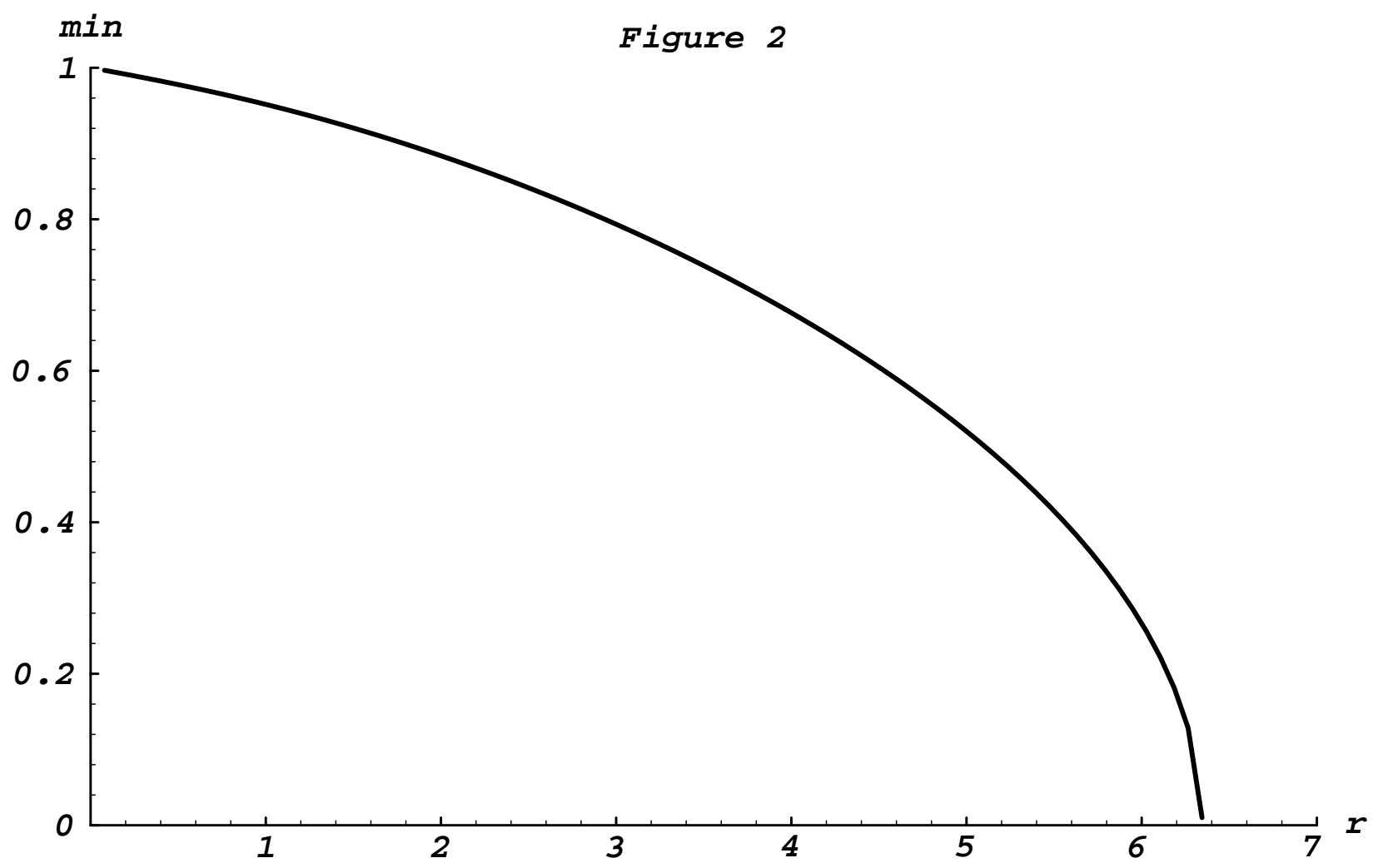




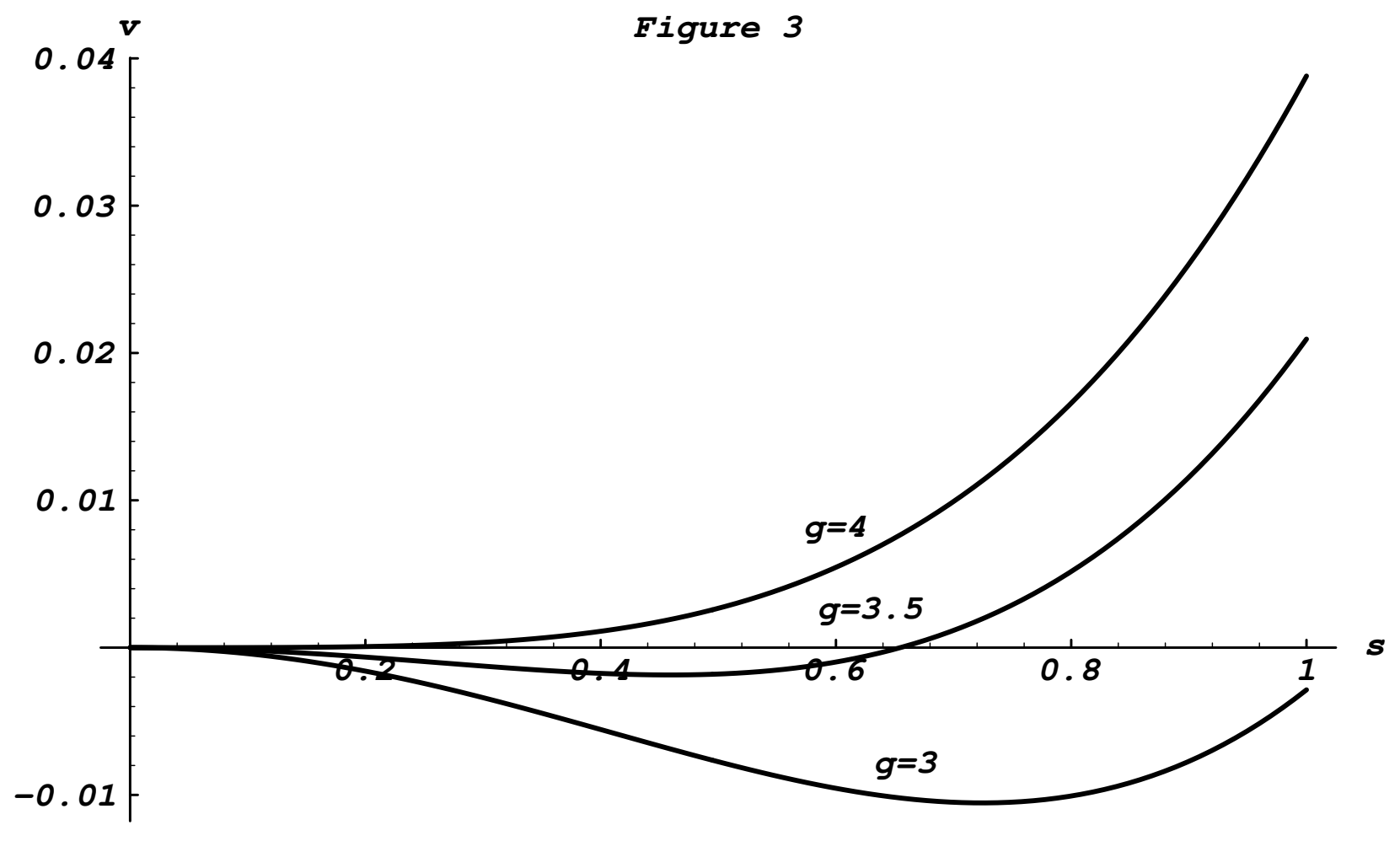




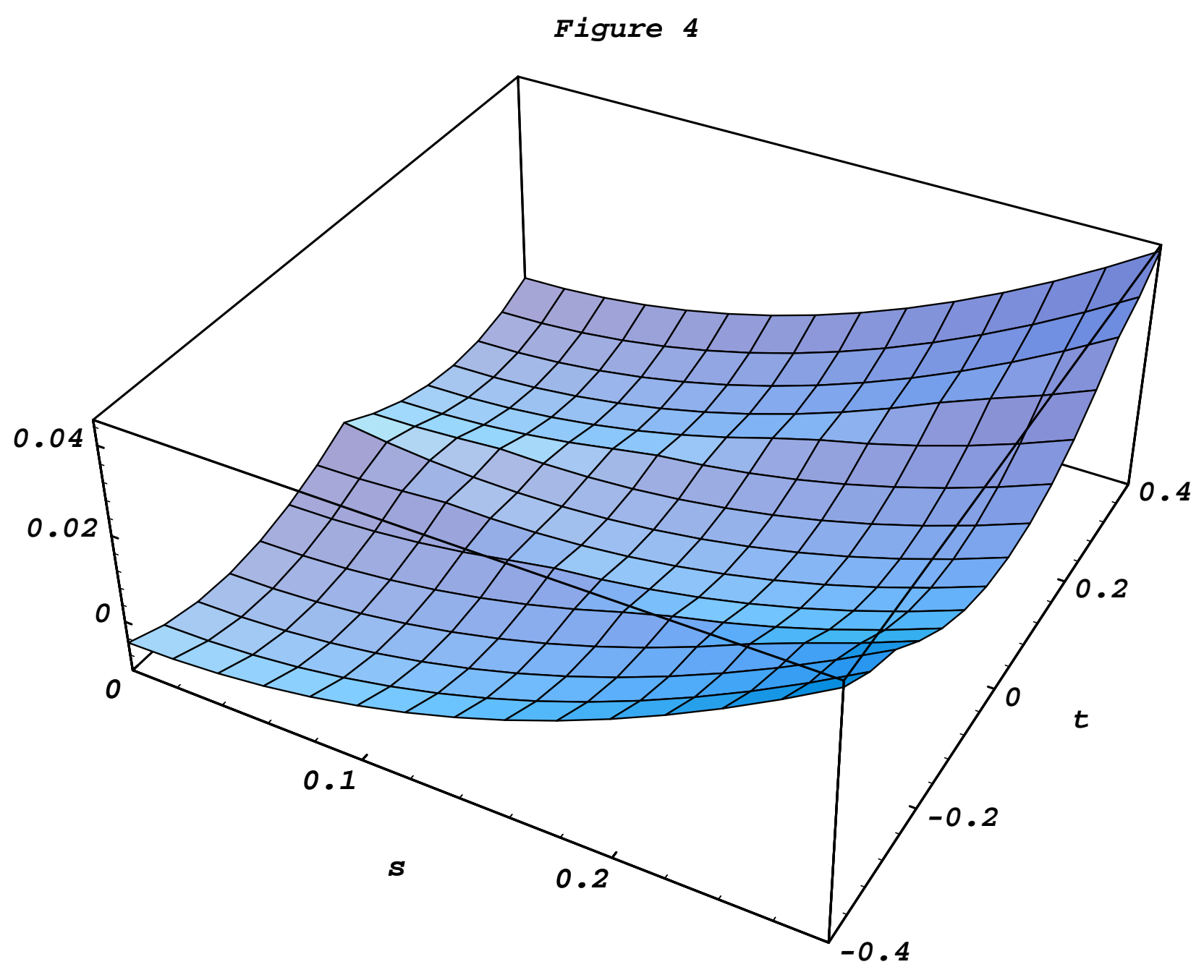




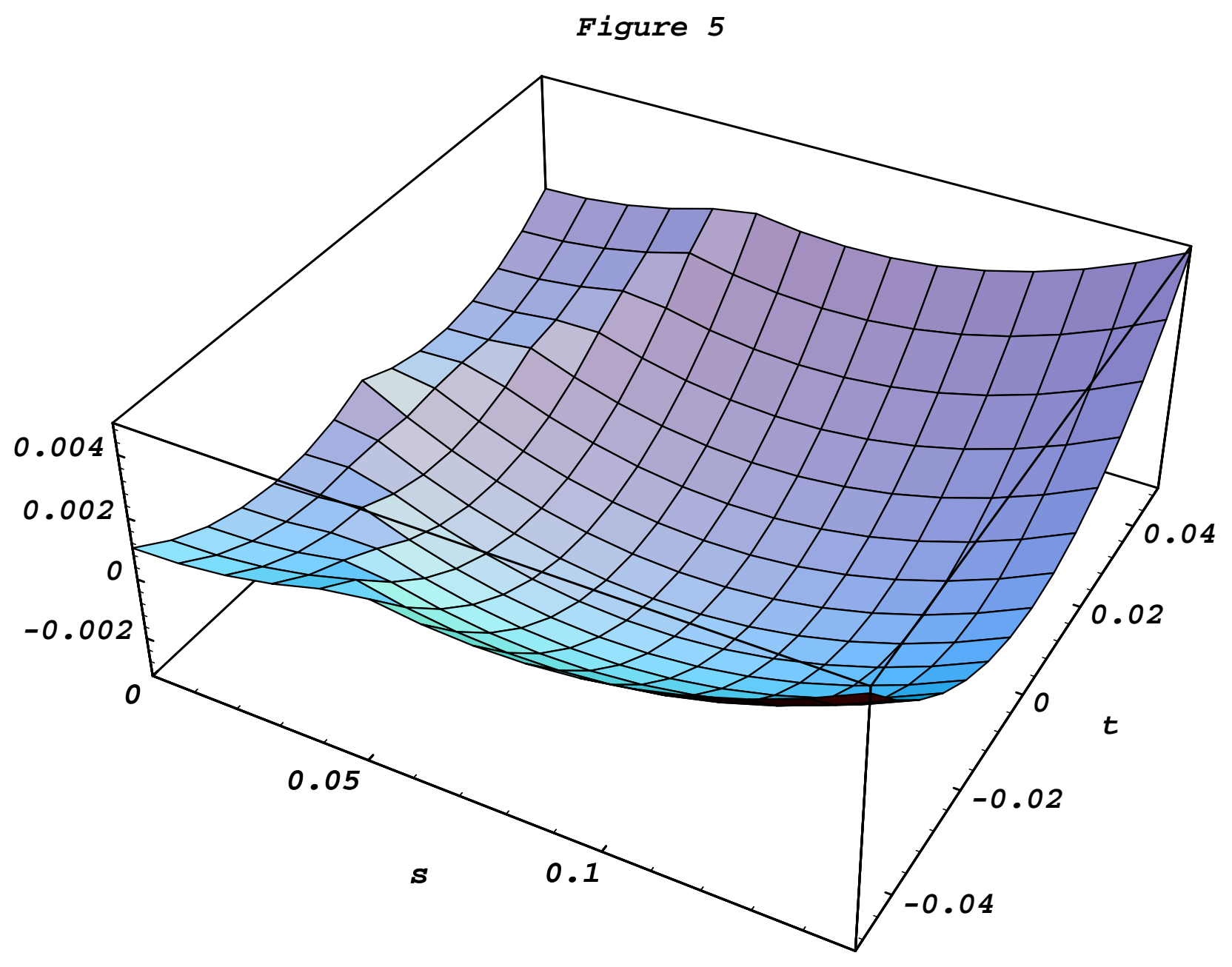

